\title{
SURFACES IN THE GRASSMANN VARIETY $G(1,3)$
}

\author{
AIGLI PAPANTONOPOULOU
}

Abstract. Let $G$ be the Grassmannian $G(1,3), Y$ a nonsingular subvariety of $G$ and $N_{Y / G}$ the normal bundle of $Y$ in $G$. Then $N_{Y / G}$ is not an ample bundle if and only if $Y$ is a Schubert cycle.

1. Introduction. In [8] we gave a complete characterization of nonsingular curves in any Grassmannian $G(r, n)$ with ample normal bundle. In the case of $G(1,3)$ we showed that the Schubert cycle of dimension 1 , which is a line, is the only nonsingular curve with normal bundle not ample. In this paper, using the intersection theories developed in [1] and in [9] we show that in dimension 2 also the Schubert cycles, which are isomorphic to $\mathbf{P}^{2}$, are the only nonsingular surfaces with normal bundle not ample. In the case of codimension 1 the normal bundle is always ample because the $\operatorname{Pic}(G)$ is trivial. Knowing that a nonsingular subvariety of $G(1,3)$ has an ample normal bundle we can apply on it several well-known theorems: (a) A vanishing theorem on formal schemes [4, Theorem 4.1], (b) A theorem on meromorphic functions [5, Chapter 6], (c) Results on the cohomological dimension of a projective variety minus a subvariety [5, Chapter 7].

Throughout the paper we are working over an algebraically closed field of characteristic 0 .

2. Preliminaries. Let $G=G(1,3)$ be the Grassmannian parametrizing $\mathbf{P}^{1}$ spaces in a fixed $\mathbf{P}^{3}$. Then $\operatorname{dim} G=4$ and $G$ is a quadric in $\mathbf{P}^{5}$. The Schubert cycles of $G$ can be described as follows:

(a) $Z_{0}=G \cap H, H$ a hyperplane in $\mathbf{P}^{5}, \operatorname{dim} Z_{0}=3$,

$$
Z_{0}=\left\{\mathbf{P}^{1} \subset \mathbf{P}^{3} \mid \mathbf{P}^{1} \cap \text { some fixed } \mathbf{P}^{1} \neq \varnothing\right\} \text {. }
$$

(b) $Z_{1}=\left\{\mathbf{P}^{1} \subset \mathbf{P}^{3} \mid \mathbf{P}^{1} \subset\right.$ some fixed $\left.\mathbf{P}^{1} \subset \mathbf{P}^{3}\right\} \cong \mathbf{P}^{2}$

$$
Z_{2}=\left\{\mathbf{P}^{1} \subset \mathbf{P}^{3} \mid \text { some fixed point } p \in \mathbf{P}^{1} \subset \mathbf{P}^{3}\right\} \cong \mathbf{P}^{1} \text {. }
$$

(c) $Z_{3}=\left\{\mathbf{P}^{1} \subset \mathbf{P}^{3} \mid\right.$ some fixed point $p \in \mathbf{P}^{1} \subset$ some fixed $\left.\mathbf{P}^{2} \subset \mathbf{P}^{3}\right\} \cong \mathbf{P}^{1}$, and for any given $Z_{3}$ there are exactly two cycles $Z_{1}$ and $Z_{2}$ such that $Z_{3}=Z_{1} \cap Z_{2} \subset G$.

Recall that on $G$ we have the short exact sequence

$$
0 \rightarrow E \rightarrow 0_{G}^{4} \rightarrow Q \rightarrow 0,
$$

where $Q$ and $E$ are the canonical bundles on $G$. The tangent bundle

Received by the editors July 12, 1978 and, in revised form, November 23, 1978.

AMS (MOS) subject classifications (1970). Primary 14M15; Secondary 14J99, 14 F05. 
$T_{G}=Q \otimes \check{E}$, therefore for all nonsingular subvarieties of $G$ the normal bundle $N_{Y / G}$ is generated by global sections. Hence by [2 Proposition 2.1] and [8, Chapter 2] if $N_{Y / G}$ is not ample then there exists a curve $C$ in $Y$ and a surjective map

$$
N_{Y / G} \rightarrow 0_{C} \rightarrow 0 .
$$

We recall that for $Y$ locally a complete intersection $N_{Y / G}$ is defined as Hom $_{0_{Y}}\left(I_{Y} / I_{Y}^{2}, 0_{Y}\right)$, where $I_{Y}$ is the sheaf of ideals defining $Y$ in $G$, and when $Y$ is nonsingular $N_{Y / G}$ is simply the usual geometric normal bundle of $Y$ in $G$.

Finally one more property of $G$ that we need is the fact that the Chow ring $A(G)$ of cycles mod rational equivalence is generated by the Schubert cycles. For example a surface $Y \neq Z_{i}, Y \sim a Z_{1}+b Z_{2}$ where $a$ and $b$ some positive integers, and $Z_{1} \cdot Z_{2}=0, Z_{i}^{2}=1$ in $A(G)$.

We use in the proof the definition and properties of ample vector bundles which are shown in [3]. We also use repeatedly the following proposition from [8]:

Proposition 2.4. Let $Y$ be a subvariety of $G(r, n)$. Then $T_{G \mid Y}$ is not ample if and only if there is a curve $C$ in $Y$ which lies in some $Z_{3}$.

In the proof of the theorem we will be using the following notation and diagrams:

$Y=$ a nonsingular surface in $G(1,3)=G, Z=$ a Schubert cycle in $G$ of $\operatorname{dim} 2$, and $D=Y \cap Z$.

$G^{\prime}=$ the blow up of $G$ with center $Y, p: G^{\prime} \rightarrow G$ the projection,

$Y^{\prime}=\mathbf{P}\left(\check{N}_{Y / G}\right), Z^{\prime}=$ the strict transform of $Z$, i.e. the blow up of $Z$ with center $D$, and $D^{\prime}=Y^{\prime} \cap Z^{\prime}$.

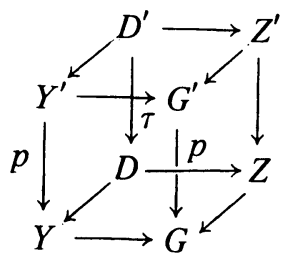

$G^{\prime \prime}=$ the blow up of $G$ with center $Z, q: G^{\prime \prime} \rightarrow G$ the projection,

$Z^{\prime \prime}=\mathbf{P}\left(\check{N}_{Z / G}\right), Y^{\prime \prime}=$ the strict transform of $Y$, i.e. the blow up of $Y$ with center $D$, and $D^{\prime \prime}=Y^{\prime \prime} \cap Z^{\prime \prime} \cong D^{\prime}$.

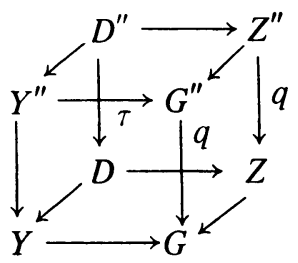


On $Y^{\prime}$ we have the canonical sequence

$$
0 \rightarrow 0_{Y^{\prime}}(-1) \rightarrow p^{*}\left(N_{Y / G}\right) \rightarrow F \rightarrow 0
$$

and restricted to $D^{\prime}$ we get

$$
0 \rightarrow N_{D^{\prime} / Z^{\prime}} \rightarrow p^{*}\left(N_{Y / G}\right)_{\mid D^{\prime}} \rightarrow F_{\mid D^{\prime}} \rightarrow 0 \text {. }
$$

Also from the canonical sequence on $Z^{\prime \prime}$ restricted to $D^{\prime \prime}$ we get

$$
0 \rightarrow N_{D^{\prime \prime} / Y^{\prime \prime}} \rightarrow q^{*}\left(N_{Z / G}\right)_{\mid D^{\prime \prime}} \rightarrow E_{\mid D^{\prime \prime}} \rightarrow 0 .
$$

From [1] we have that in the Chow ring $A(D)$ the intersection cycle is given by $Y \cdot Z=\tau_{*} c_{1}\left(F_{\mid D^{\prime}}\right)=\tau_{*} c_{1}\left(D_{\mid D^{\prime \prime}}\right)$.

\section{Nonsingular surfaces in $G(1,3)$.}

THEOREM. Let $Y$ be a nonsingular surface in $G=G(1,3) . N_{Y / G}$ the normal bundle of $Y$ in $G$ is not ample if and only if $Y=Z_{i}$ (a Schubert cycle of $\left.\operatorname{dim} 2\right)$.

Proof. If $Y=Z_{i}$ then $T_{G \mid Y}=T_{Y} \oplus Q_{\mid Y}$ or $T_{G \mid Y}=T_{Y} \oplus \check{E}_{\mid Y}$. By [8, 2.2 and 2.3]

$$
Q_{\mid Z_{3}} \cong \check{E}_{\mid Z_{3}} \cong 0_{Z_{3}}(1) \oplus 0_{Z_{3}}
$$

where $Z_{3} \cong \mathbf{P}^{1}$. Hence $N_{Y / G}$ restricted to a cycle of type $Z_{3}$ is not ample, therefore $N_{Y / G}$ is not ample.

Now assume that $Y \neq Z_{i} . N_{Y / G}$ is a vector bundle on $Y$ which is generated by its global sections, hence by [2. Proposition 2.1] it is sufficient to show that $N_{Y / G \mid C}$ is ample for every curve $C$ in $Y$. By [8, Proposition 2.4] $T_{G \mid C}$ is not ample if and only if $C=Z_{3} \cong \mathbf{P}^{1}$. Since $N_{Y / G \mid C}$ is a quotient bundle of $T_{G \mid C}$, it will be sufficient to show that for any cycle $Z \subset G, N_{Y / G \mid D}$ is ample, where $D=Y \cap Z$. Let $I_{D}$ be the sheaf of ideals defining $D=Y \cap Z$ in $Y$. Then

$$
\tau^{*}\left(I_{D}\right) \oplus 0_{D^{\prime \prime}}=\check{N}_{D^{\prime \prime} / Y^{\prime \prime}}
$$

For any curve $C$ in $D^{\prime \prime}$ not in any fiber the map $\tau$ is of degree 1 and $\tau_{*}\left(\check{N}_{D^{\prime \prime} / Y^{\prime \prime} \mid C}\right)=\check{N}_{C / Y}$. For the rest of the proof let $C$ be a cycle of type $Z_{3}$. Since $Y \neq Z_{i}$, $\operatorname{deg}_{G} Y>1$ and $(C \cdot C)_{Y}<1$. Hence $\tau_{*}\left(N_{D^{\prime \prime} / Y^{\prime \prime} \mid C}\right)=0_{C}(v)$ where $v<1$, and the exact sequence (2) restricted to $C$ becomes

$$
0 \rightarrow 0_{C}(v) \rightarrow q^{*}\left(N_{Z / G}\right)_{\mid C} \rightarrow E_{\mid C} \rightarrow 0 .
$$

The vector bundle

$$
q^{*}\left(N_{Z / G}\right)_{\mid C}=0_{C}(1) \oplus 0_{C}
$$

therefore $E_{\mid C}$ is an ample line bundle on $C$. Hence the line bundle $E_{\mid D^{\prime \prime}}$ has a global section with zeroes on $C$. Since $\tau_{*} c_{1}\left(F_{\mid D^{\prime}}\right)=\tau_{*} c_{1}\left(E_{\mid D^{\prime \prime}}\right)$ we get a global section of the line bundle $F_{\mid \mathbf{D}^{\prime}}$ which is not constant on $C$. Hence $F_{\mid C}$ is an ample line bundle on $C$. Consider now the exact sequence (1). $\tau_{*}\left(N_{D^{\prime} / Z^{\prime} \mid C}\right)=$ $N_{C / Z}$ where $C \cong \mathbf{P}^{1}$ and $Z \cong \mathbf{P}^{2}$. Hence $\tau_{*}\left(N_{D^{\prime} / Z^{\prime} \mid C}\right)=0_{C}(1)$ and $N_{Y / G \mid C}=$ $0_{C}(u) \oplus 0_{C}(w)$ where $u>0$ and $w>0$. Hence $N_{Y / G \mid D}$ is ample. Q.E.D. 


\section{BIBLIOGRAPHY}

1. W. Fulton and R. MacPherson, Intersecting cycles on an algebraic variety, Aarhus Univ., Aarhus, 1976 (preprint).

2. D. Gieseker, p-ample bundles and their Chern classes, Nagoya Math. J. 43 (1971), 91-116.

3. R. Harshorne, Ample vectors bundles, Inst. Hautes Etudes Sci. Publ. Math., No. 29 (1966), 63-94.

4. ___ Ample subvarieties of algebraic varieties, Lecture Notes in Math., vol. 156, SpringerVerlag, Berlin and New York, 1970.

5. Cohomological dimension of algebraic varieties, Ann. of Math.(2) 88 (1968), 403-450.

6. __ Ample vector bundles on curves, Nagoya Math. J. 43 (1971), 71-89.

7. V. D. Hodge and D. Pedoe, Methods of algebraic geometry. Vols. I, II, III, Cambridge Univ. Press, 1947, $1952,1954$.

8. A. Papantonopoulou, Curves in Grassmann varieties, Nagoya Math. J. 66 (1977), 121-137.

9. (1977), 80-93.

Department of Mathematics, Bryn Mawr College, Bryn Mawr, Pennsylvania 19010 